\title{
How solar radiation pressure acts on RSAT and VSAT with a small evolving tip-off in SELENE
}

\author{
Jinsong Ping ${ }^{1,3}$, Arata Sengoku ${ }^{4}$, Nobuaki Nagaoka ${ }^{5}$, Takahiro Iwata ${ }^{6}$, Koji Matsumoto ${ }^{2}$, and Nobuyuki Kawano ${ }^{1,2}$ \\ ${ }^{1}$ Department of Astronomical Science, the Graduate University for Advanced Studies, 2-22-1 Osawa, Mitaka, Tokyo 181-8588, Japan \\ ${ }^{2}$ Division of Earth Rotation, National Astronomical Observatory, 2-12 Hoshigaoka Machi, Mizusawa, Iwate 023-0861, Japan \\ ${ }^{3}$ Department of Astronomy, Beijing Normal University, 19 Xinjiekou Waidajie, Haidian, Beijing 100875, China \\ ${ }^{4}$ Hydrographic Department, Maritime Safety Agency, Tsukiji 5-3-1, Chuo-ku, Tokyo 104-0045, Japan \\ ${ }^{5}$ Department of Space Technique, Toshiba Co., 1 Komukai-Toshiba-Cho, Saiwai-Ward, Kawasaki-City 210, Japan \\ ${ }^{6}$ Tsukuba Space center, National Space Development Agency, 2-1-1 Sengen, Tsukuba, Ibaraki 305-8505, Japan
}

(Received October 13, 2000; Revised June 26, 2001; Accepted June 28, 2001)

\begin{abstract}
The solar radiation pressure model for the sub-satellites RSAT and VSAT in the SELENE project is improved to correct the mean acceleration due to an evolving tip-off of the spin during the life time of satellites. The shape of the satellites is assumed to be a regular octagonal pillar. Solar radiation pressure force components acting on each surface element of the satellite are calculated independently and summed vectorially during a total period of Euler's free nutation of the satellite to obtain the mean acceleration of the satellite center of mass. The Doppler tracking data reduction process for the RSAT is simulated after incorporating the modified model into the orbit analysis software. Comparing with two other types of solar radiation pressure models, the standard cannonball model and a non tip-off model, it is found that when the tip-off becomes larger than $5^{\circ} / \mathrm{s}$, the orbit determination result of using the modified model is better than the results of using other ones.
\end{abstract}

\section{Introduction}

In the summer of 2004, a Japanese lunar exploration mission, SELENE (SELenologial and ENgineering Explorer), will be injected into polar orbit(s) around the Moon. One of its main scientific targets is to directly measure the gravity field in both near- and far-side of the Moon (Namiki et al., 1999). This is the basic difference from the historical missions of lunar gravity research. Three spacecrafts $(\mathrm{S} / \mathrm{C})$, a main lunar orbiter, a relay sub-satellite (RSAT) and a free-flying VLBI radio source (VSAT), constitute the mission of SELENE. RSAT and VSAT are two spin stabilized sub-satellites with a normal spin rate of $15 \mathrm{rpm}$. The three satellites will be tracked by using different methods, i.e., 2-way Doppler tracking, 4-way satellite-to-satellite tracking (hi-lo SST with ground station in the loop, much like TDRSS) between the Earth, RSAT and the main orbiter, and the differential VLBI observation between RSAT, VSAT and quasars. Specifically, by using SST method (e.g. Lambeck, 1988), the gravity field of the lunar far-side can be mapped directly. The orbiting software package, GEODYN II of NASA/GSFC (Pavlis et al., 1998) will be used for data analysis and orbit determination.

In SELENE, SST is the key tracking technique for measuring the far-side lunar gravity variation. The 4-way Doppler up-link signal transmitted from the ground station will be locked and forwarded by RSAT to the main orbiter. RSAT bridges the link between tracking station and the main

Copy right (C) The Society of Geomagnetism and Earth, Planetary and Space Sciences (SGEPSS); The Seismological Society of Japan; The Volcanological Society of Japan; The Geodetic Society of Japan; The Japanese Society for Planetary Sciences. orbiter. For the orbits of lunar orbiters are perturbed by the lunar anomalous gravity field, the line-of-sight Doppler measurements or the long-term orbital variations of the lunar orbiters are used to map the lunar gravity field. To estimate the lunar gravity field more precisely, the accuracy of the orbits needs to be improved for these lunar orbiters, especially for RSAT. It also has been proven that the surface forces acting on the satellites are non-negligible error sources for precise orbit determination (Sengoku et al., 1995). Floberghagen et al. (1999) developed a force model for the lunar albedo effect on low lunar orbiters. In this article, the solar radiation pressure acting on RSAT and VSAT is discussed. The shape and mass of RSAT and VSAT are designed to be identical. They will evolve in the same orbit plane with same attitude before and after the separation from the main orbiter. Therefore, the solar radiation pressure model(s) for both $\mathrm{S} / \mathrm{C}$ can be assumed to be identical as well.

A model has been developed by Kubo-oka and Sengoku (1999) to describe the solar radiation pressure acting on a spin stabilized RSAT with a shape of regular octagonal pillar plus a docking column, without any nutation or tip-off being considered when the $\mathrm{S} / \mathrm{C}$ is inserted into its orbit. This model is called non tip-off model hereafter. However, after the publication of the non tip-off model, the shape design of RSAT and VSAT is modified. The docking assemblies, the separation system between RSAT/VSAT and the main orbiter will be installed on the main orbiter rather than on RSAT/VSAT. The columns corresponding to the docking assemblies will therefore not be included in the shape of 


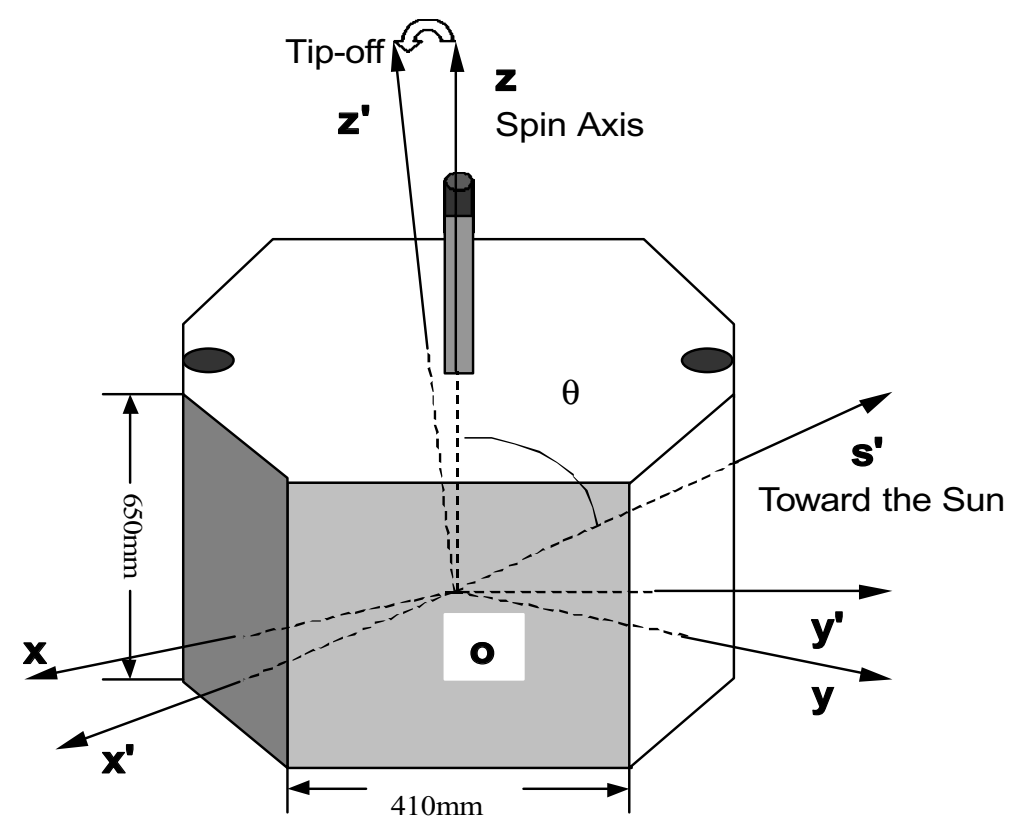

Fig. 1. Shape and satellite fixed coordinate systems of RSAT and VSAT.

RSAT/VSAT. In a simulation research of SELENE 4-way Doppler tracking, a free nutation is noticed during the $\mathrm{S} / \mathrm{C}$ spin. This nutation is induced by the tiny offset of satellite mass away from the uniform distribution (Ping et al., 2001). As a rigid body, the nutation period, 72 seconds of RSAT is defined by the diagonal elements of the matrix in Eq. (1), and the spin rate of $90^{\circ} / \mathrm{s}$. Ping et al. (2001) also studied the effect on Doppler tracking due to a small tip-off of angular velocity of $5^{\circ} / \mathrm{s}$, which is produced by the separating process of RSAT/VSAT from the main orbiter. The tip-off, which has been found in the RSAT/VSAT separation experiments on ground, will enlarge the amplitude of S/C nutation but will not change the nutation period. In the worst case, after one year evolution in the orbit, it will be increased to about twice of the initial value by the lunar gravity field and the surface forces acting on S/C. Due to the tip-off, the actual solar pressure acting on RSAT/VSAT should be different from the case of non tip-off. Taking into account this kind of difference, the non tip-off solar radiation pressure model is modified to correct the extra effect induced by a small tip-off of RSAT/VSAT.

\section{Average Solar Radiation Pressure during the Period of Free Euler Nutation}

Two types of satellite fixed coordinate systems are adopted in the calculation for RSAT and VSAT. See Fig. 1. One is denoted o-xyz, the mass center fixed system, which shares the orbital movement of the $\mathrm{S} / \mathrm{C}$ as a mass point only; another is denoted o-x'y'z', the satellite body fixed system, which shares the whole movement of the satellite as a rigid body in the inertial space. Before and during the period when the satellite is separated from the main orbiter, the two systems coincide. In the o-xyz system, the $z$-axis is chosen to be parallel to the spin axis, $\mathrm{x}$-axis as $\mathbf{x}=\mathbf{s} \times \mathbf{z}, \mathrm{y}$-axis as $\mathbf{y}=\mathbf{z} \times \mathbf{x}$, where $\mathbf{s}$ is the direction from the mass center of the spacecraft towards the Sun. Differences between the two systems after the separation include the satellite spin, the Euler free nutation of the rigid body, and a small tip-off following with the spin (Macmillan, 1936). Three Euler angles fully describe the transformation between the two systems at any given time after separation.

In this paper, compared to Kubo-oka and Sengoku (1999), the main assumptions and differences are, besides the docking assemblies, both spin and tip-off are considered; the orientation of satellite spin axis changes with the periods of the satellite spin and the nutation; the orientation of o-xyz coordinate system does not change, where the $z$-axis is termed the mean spin axis; in the o-xyz system, the angle between the $\mathrm{z}$-axis and the direction towards the Sun is denoted as $\theta$. In the simulation it is found that the docking system contributes little to the total solar radiation pressure. The tip-off, however, always induces an extra force by the solar radiation pressure, compared to the non tip-off case. The satellite mass $m$ is $39.0 \mathrm{~kg}$, the moment of inertia $\mathbf{I}$ of RSAT and VSAT in unit of $\mathrm{kg} \cdot \mathrm{m}^{2}$ is, in the o-X'y'z' system

$$
\mathbf{I}=\left[\begin{array}{ccc}
3.805 & 5.074 \times 10^{-4} & -8.919 \times 10^{-5} \\
5.074 \times 10^{-4} & 3.607 & -5.310 \times 10^{-2} \\
-8.919 \times 10^{-5} & -5.310 \times 10^{-2} & 3.609
\end{array}\right]
$$

Therefore a set of Euler's differential equations may be established in the o-xyz system as

$$
\mathbf{I} \dot{\omega}=\mathbf{N}-\omega \times \mathbf{I} \omega,
$$

where, $\boldsymbol{\omega}=\left(\omega_{1}, \omega_{2}, \omega_{3}\right)$ is $\mathrm{S} / \mathrm{C}$ spin vector in the coordinate frame fixed on the satellite; $\mathbf{N}$ is moment of the exterior forces which is assumed to be $\mathbf{0}$ in following calculation. Then, the relations between $\boldsymbol{\omega}$ and the three Euler angles $\theta$, $\psi$ and $\phi$ in the space are 


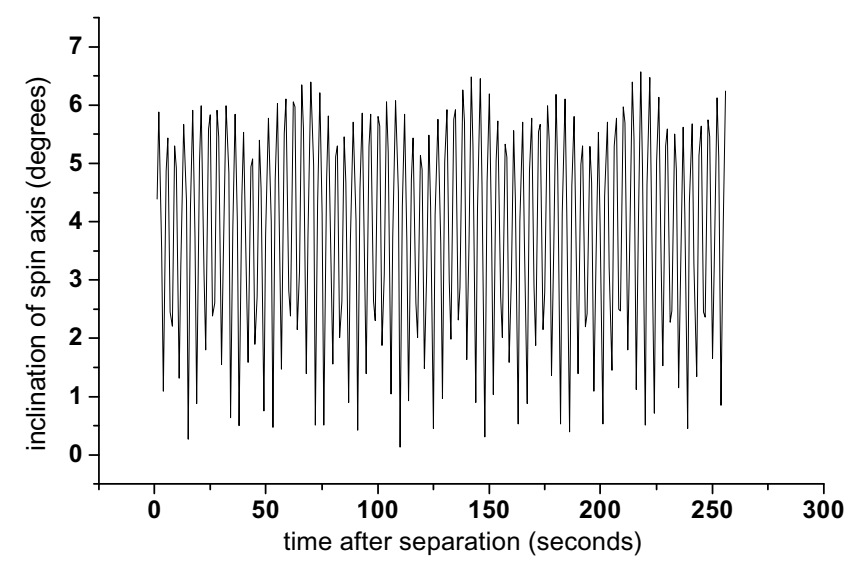

Fig. 2. Inclination of spin axis after satellite separation for RSAT and VSAT.

$$
\left\{\begin{aligned}
\dot{\theta} & =\omega_{2} \cos \phi-\omega_{3} \sin \phi \\
\dot{\psi} \sin \theta & =\omega_{2} \sin \phi+\omega_{3} \cos \phi \\
\dot{\phi} & =-\left(\omega_{2} \sin \phi+\omega_{3} \cos \phi\right) \cot \theta+\omega_{1}
\end{aligned}\right.
$$

where $\dot{\theta}, \dot{\psi}$ and $\dot{\phi}$ are the time derivatives of $\theta, \psi$ and $\phi$, respectively (Macmillan, 1936). Solving Euler's differential equations, the evolution of the inclination of $z$ '-axis related to z-axis after separation can be obtained. This result is shown in Fig. 2. Both the spin period and the nutation period can easily be recognized.

For RSAT and VSAT, the following assumptions are adopted, for reasons of simplicity: i) The shape of $\mathrm{S} / \mathrm{C}$ is a regular octagonal pillar. Because the antennas' surfaces are very small, the contributions of the antennas are neglected. ii) The $\mathrm{S} / \mathrm{C}$ spin periods of $\omega_{3}=60 \sim 120^{\circ} / \mathrm{s}$ do not give obvious difference in the result, a value of $90^{\circ} / \mathrm{s}$ is adopted; in the worst case, the initial tip-off angular velocity of $\omega_{1}=5^{\circ} / \mathrm{s}$ at the $\mathrm{S} / \mathrm{C}$ separation epoch is assumed, it will be increased to $\sim 10^{\circ} / \mathrm{s}$ after one year orbital evolution. iii) The solar radiation pressure is averaged during the interval of Euler's nutation period of $\sim 72$ seconds. This period, which is defined by $\omega_{3}$ and $\mathbf{I}$, is much shorter than the orbital period of $\sim 4$ hours. iv) The z-axis of the o-xyz system is assumed to be normal to the lunar orbital plane at the time of orbit insertion. v) The optical properties of each surface element can be expressed by a linear combination of a black body, a perfect mirror and a Lambert diffuser (Milani et al., 1987). vi) The surface materials are chosen as solar cells for side panels, and black kapton for top and bottom panels. Their reflectivities are given in Table 1.

At given time $t$, the total solar radiation pressure $\mathbf{F}(t)$ in o-xyz coordinate system is,

$$
\mathbf{F}(t)=\sum_{i=1}^{k} d \mathbf{F}_{i}(t),
$$

for each flat area element, $\mathbf{F}_{i}(t)$ can be expressed as (Milani et al., 1987)
Table 1. Specular and diffuse reflectivity of the surface materials for RSAT.

\begin{tabular}{cccc}
\hline Material & Area $\left(\mathrm{m}^{2}\right)$ & $\rho$ & $\delta$ \\
\hline Solar panel & $.2296^{*}$ & 0.03 & 0.21 \\
Black kapton & $.8119^{*}$ & 0.00 & 0.86 \\
\hline
\end{tabular}

* for each panel or plate.

$$
\begin{aligned}
d \mathbf{F}_{i}(t)= & -\frac{A_{i} \Phi(t)}{c}\left[\left(1-\rho_{i}\right) \mathbf{s}\right. \\
& \left.+2\left(\rho_{i} \mathbf{n}_{i}(t) \cdot \mathbf{s}+\frac{\delta_{i}}{3}\right) \mathbf{n}_{i}(t)\right] \mathbf{n}_{i}(t) \cdot \mathbf{s},
\end{aligned}
$$

where $c=299792458 \mathrm{~m} / \mathrm{s}, \Phi(t), A_{i}, \rho_{i}, \delta_{i}$ and $\mathbf{n}_{i}(t)$ are the velocity of light in vacuum, the flux of solar radiation at position of $\mathrm{S} / \mathrm{C}$, the area of $i$ th flat plate, the specular reflectivity, the diffuse reflectivity, and the unit normal vector of the plate, respectively. $A_{i}, \rho_{i}$ and $\delta_{i}$ are listed in Table 1. The $\mathbf{n}_{i}(t)$ can be obtained after transforming from $\mathbf{n}_{i}(0)$ by rotating $\mathbf{n}_{i}(0)$ about three Euler's angles with respective to certain axes. The three Euler's angles can be solved by integrating Eqs. (2) and (3) from 0 to $t$. The mean force of solar radiation pressure $\mathbf{F}$ during one period of Euler nutation $T$ is:

$$
\mathbf{F}=\frac{1}{T} \int_{0}^{T} \sum_{i=1}^{k} d \mathbf{F}_{i}(t) d t .
$$

Employing the above equations and parameters, using different value of tip-off separately, the mean solar radiation pressure of different $\theta$ in the o-xyz system on RSAT or VSAT can be obtained. As examples, the results of the solar radiation pressures parallel to the $\mathrm{x}, \mathrm{y}$ and $\mathrm{z}$-axes, separately obtained from the tip-off of $5 \% \mathrm{~s}$ and of $10 \%$, are shown in Figs. 3(a), (b) and (c), respectively. It is noticed that these results are different from the non tip-off case discussed by Kubo-oka and Sengoku (1999), which are also shown in the same figures.

\section{Differences in Solar Radiation Pressure be- tween the Models with and without the Evolving Tip-Off Angle}

From Eq. (5), Kubo-oka and Sengoku (1999) have deduced a mean solar radiation pressure model for RSAT. In their work, tip-off is not taken into account, the average of acceleration is obtained in one spin period, and the spin axis orientation is assumed not to change after orbit insertion. Under these assumptions, the solar radiation pressure model is given as

$$
\left(\mathbf{a}_{\text {total }}\right)^{\text {non tip-off }}=\mathbf{a}_{\text {oct }}+\mathbf{a}_{\mathrm{tb}} .
$$

where $\mathbf{a}_{\mathrm{oct}}$ and $\mathbf{a}_{\mathrm{tb}}$ are components of acceleration due to the solar radiation acting on the solar cells surface, and on the top and bottom surface of the pillar. They read

$$
\begin{array}{r}
\left(\mathbf{a}_{\text {total }}\right)_{\mathrm{x}}^{\text {non tip-off }}=\left(\mathbf{a}_{\mathrm{oct}}\right)_{\mathrm{x}}=\left(\mathbf{a}_{\mathrm{tb}}\right)_{\mathrm{x}}=0\left[\mathrm{~m} / \mathrm{sec}^{2}\right], \\
\left(\mathbf{a}_{\mathrm{oct}}\right)_{\mathrm{y}}=-\frac{8 A_{\mathrm{oct}} \Phi(t)}{3 m c} \sin \theta\left\{\frac{3+\rho_{s}}{\pi} \sin \theta+\frac{\delta_{s}}{2}\right\},
\end{array}
$$



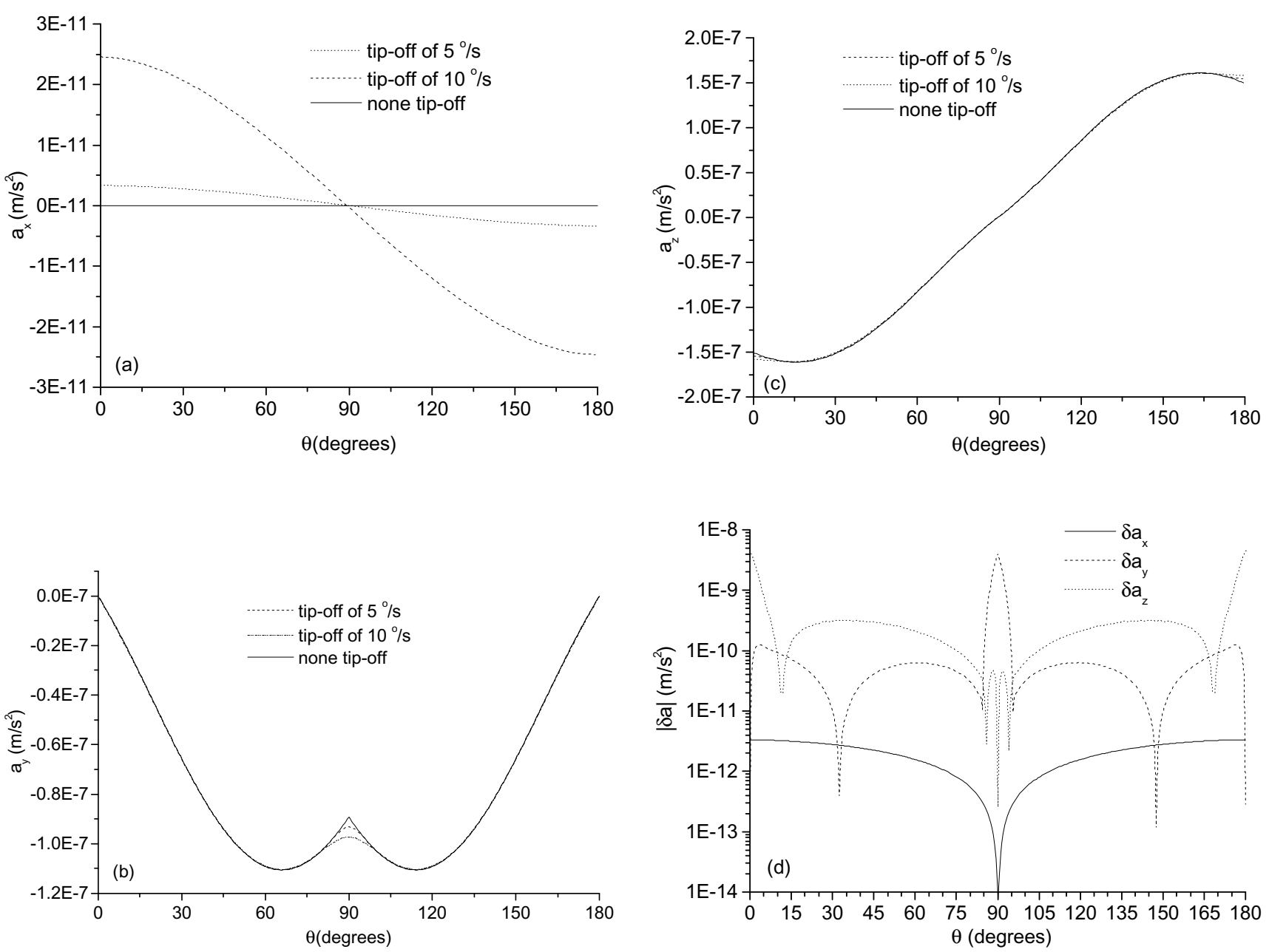

Fig. 3. Mean acceleration due to the solar radiation pressure in the o-xyz coordinate system: (a), (b) and (c) are components parallel to the $\mathrm{x}$, $\mathrm{y}$ and $\mathrm{z}$-axis, respectively; (d) shows the differences between non tip-off and a tip-off of $5^{\circ} / \mathrm{s}$ in the three directions.

$$
\begin{gathered}
\left(\mathbf{a}_{\mathrm{oct}}\right)_{\mathrm{z}}=-\frac{8 A_{\mathrm{oct}} \Phi(t)}{\pi m c}\left(1-\rho_{s}\right) \sin \theta \cos \theta, \\
\left(\mathbf{a}_{\mathrm{tb}}\right)_{\mathrm{y}}=-\frac{A_{\mathrm{tb}} \Phi(t)}{m c}\left(1-\rho_{g}\right) \sin \theta|\cos \theta|, \\
\left(\mathbf{a}_{\mathrm{tb}}\right)_{\mathrm{z}}=-\frac{A_{\mathrm{tb}} \Phi(t)}{m c} \cos \theta\left\{\left(1+\rho_{g}\right)|\cos \theta|+\frac{2}{3} \delta_{g}\right\} .
\end{gathered}
$$

where, $\rho_{s}, \delta_{s}, \rho_{g}$ and $\delta_{g}$ denote the specular reflectivities and the diffuse reflectivities for the solar panel (subscript $s$ ) and black capton (subscript $g$ ), respectively. $A_{\text {oct }}$ and $A_{\text {tb }}$ are area components of the solar cells surface and top or bottom plate, respectively.

In the case of non tip-off, the dependencies of the accelerations on $\theta$ in the o-xyz system due to the solar radiation pressure are also shown in Figs. 3(a), (b) and (c). The corresponding differences between the results from a tip-off of $5 \%$ and from non tip-off model are given in Fig. 3(d).

In Fig. 3 it can be seen that the maximum solar radiation pressure at the direction parallel to the $\mathrm{x}$-axis is $2.5 \times 10^{-11}$ $\mathrm{m} / \mathrm{s}^{2}$ for the tip-off of $10.0^{\circ} \mathrm{s}$. It is too small to be taken into account for present applications, and can be neglected. At the direction parallel to the z-axis, the maximum difference of $5 \times 10^{-9} \mathrm{~m} / \mathrm{s}^{2}$ appears at $\theta=0^{\circ}$ and $180^{\circ}$. Due to a restriction of the middle gain antenna power pattern of RSAT, $\left|\theta-90^{\circ}\right|>20^{\circ}$, tracking data of RSAT are likely to be useless because of the poor transmission gain or the received signal to noise ratio. The orientation of the mean spin axis is assumed to be normal to the lunar orbital plane. According to Kubo-oka and Sengoku (1999), $\theta$ changes sinusoidally between $\sim 85^{\circ}$ and $\sim 95^{\circ}$ during its life time after the orbit insertion. In this range, the maximum difference of the z-component is only about $5 \times 10^{-11} \mathrm{~m} / \mathrm{s}^{2}$, and the mean solar radiation pressure model in the non tip-off case can be used in the case of tip-off. At the direction parallel to the y-axis, for the tip-off of $5 \%$ s, the maximum difference of $4 \times 10^{-9} \mathrm{~m} / \mathrm{s}^{2}$ appears at $\theta=90^{\circ}$. This acceleration can produce a velocity variation of $0.4 \mathrm{~mm} / \mathrm{s}$ per day or $12 \mathrm{~mm} / \mathrm{s}$ during a month, the length of the arc for the lunar gravity field recovery from the Doppler tracking data. This bias is a little large to determine the orbit of RSAT from line of sight Doppler tracking data with an expected RMS noise of 0.2 $\mathrm{mm} / \mathrm{s}$. The solar radiation pressure model of this part needs to be improved.

\section{The New Model}

From June 3 to July 3, 2000, 26 experiments for the RSAT/VSAT separation system have been performed. 19 
Table 2. Coefficients of multiple Gauss functions and polynomial functions.

\begin{tabular}{cccccc}
\hline$X$ & $X_{\circ}$ & $b_{\circ}$ & $b_{1}$ & $b_{2}$ & $b_{3}$ \\
\hline$G_{1}$ & $2.47617 \times 10^{-3}$ & 1.00146 & 0.397307 & $3.91492 \times 10^{-2}$ & 0.0 \\
$w_{1}$ & $6.92524 \times 10^{-2}$ & 0.999106 & 0.191168 & $-1.88479 \times 10^{-3}$ & 0.0 \\
$G_{2}$ & $2.64936 \times 10^{-4}$ & 0.997164 & 0.368572 & $2.65442 \times 10^{-2}$ & $-1.55 \times 10^{-3}$ \\
$w_{2}$ & $2.24006 \times 10^{-2}$ & 0.988190 & 0.163373 & $-5.11201 \times 10^{-3}$ & 0.0 \\
$G_{3}$ & $3.57983 \times 10^{-5}$ & 0.931110 & 0.204930 & 0.0 & 0.0 \\
$w_{3}$ & $6.13342 \times 10^{-3}$ & 0.978592 & 0.098114 & $-1.11768 \times 10-2$ & $9.17 \times 10-4$ \\
\hline
\end{tabular}

spin angular velocity data and 20 tip-off velocity data have been obtained. The spin rates distribute from $71.1^{\circ} / \mathrm{s}$ to $81.3^{\circ} / \mathrm{s}$. In worst cases, tip-off velocity values are $\sim 5.0^{\circ} / \mathrm{s}$. As mentioned earlier, in the calculation, spin rates in a range from $60^{\circ} / \mathrm{s}$ to $120^{\circ} / \mathrm{s}$ are tested and compared. It is noticed that the average solar radiation pressure acting on RSAT during Euler's free nutation period should not be affected by changing the spin velocity in this range, but should be affected by different tip-off velocities. The spin rate of $90^{\circ} / \mathrm{s}$ is adopted here. Due to the solar radiation pressure acting on different sides of the RSAT surface, the amplitude of inclination of RSAT spin axis will be enlarged by twice the initial value, after having evolved in its orbit for one year. In the worst case, the tip-off of $5^{\circ} / \mathrm{s}$ at separation should become $\sim 10^{\circ} \mathrm{s}$ after one year. So the tip-off range from $0.5^{\circ} / \mathrm{s}$ to $10.5^{\circ} / \mathrm{s}$ is adopted to estimate a new solar radiation pressure model in this research.

Although the mean solar radiation pressure is different for RSAT/VSAT between the cases of tip-off and of non tip-off, an idea of modifying the non tip-off model is accepted rather than developing a new one. The reasons are: the method used by Kubo-oka and Sengoku (1999) can successfully analyze the solar radiation pressure acting on a spin stabilized $\mathrm{S} / \mathrm{C}$; and, for a small tip-off, the variation is very small. In the range of $80^{\circ} / \mathrm{s}<\theta<100^{\circ} / \mathrm{s}$, only a small correction needs to be added to the non tip-off model at the direction parallel to the $y$-axis in the o-xyz coordinate system. Other differences can be neglected without loss of accuracy. Theoretically, the solar radiation pressure model for RSAT/VSAT with tip-off velocity from $0^{\circ} / \mathrm{s}$ to $10^{\circ} / \mathrm{s}$ can be written as

$$
\begin{gathered}
\left(\mathbf{a}_{\text {total }}\right)_{\mathrm{x}, \mathrm{z}}^{\mathrm{tip} \text {-off }} \approx\left(\mathbf{a}_{\text {total }}\right)_{\mathrm{x}, \mathrm{z}}^{\text {non tip-off }}, \\
\left(\mathbf{a}_{\text {total }}\right)_{\mathrm{y}}^{\text {tip-off }}=\left(\mathbf{a}_{\text {total }}\right)_{\mathrm{y}}^{\text {non tip-off }}+\delta \mathbf{a}_{\mathrm{y}}^{\text {fit }},
\end{gathered}
$$

Based on Eqs. (13) and (14), the work of developing a new model reduces of finding a function for the small correction term $\delta \mathbf{a}_{\mathrm{y}}^{\text {fit }}$ to fit the difference at the direction parallel to the y-axis in Fig. 3(d). Various functions are introduced in the fitting calculation by the least squares adjustment. Among them, Gaussian functions can get smallest RMS of post-fit residuals. However, a single Gaussian function is not enough. After trying multiple Gaussian functions of different numbers, three Gaussian functions are found to be able to give the most optimal fitting result. Functions of numbers higher than three can hardly improve the fitting process. The RMS of post-fit residuals by using three Gaussian functions is $5.69 \times 10^{-11} \mathrm{~m} / \mathrm{s}^{2}$. The maximum residual of $6 \times 10^{-11} \mathrm{~m} / \mathrm{s}^{2}$ in the required range of $80^{\circ}<\theta<100^{\circ}$ can be neglected. It is the same for other small tip-offs of smaller than $10^{\circ} / \mathrm{s}$. Then, the term of $\delta \mathbf{a}_{\mathrm{y}}^{\mathrm{fit}}$ is written as

$$
\begin{aligned}
\delta \mathbf{a}_{\mathrm{y}}^{\mathrm{fit}}= & -\frac{A_{\mathrm{tb}} \Phi(t)}{m c}\left(1-\rho_{g}\right) \\
& \cdot \sum_{i=1}^{3} \frac{G_{i}}{\sqrt{\frac{\pi}{2}} w_{i}} \exp \left\{-\left[\frac{2\left(\theta-\frac{\pi}{2}\right)^{2}}{w_{i}^{2}}\right]\right\},
\end{aligned}
$$

the coefficient $G_{i}$ and $w_{i}$ in Gauss functions can be expressed as polynomial functions

$$
X=X_{\circ}\left[b_{\circ}+\sum_{j=1}^{3} b_{j}\left(\omega_{\text {tip-off }}-5^{\circ} / \mathrm{s}\right)^{j}\right] .
$$

where character $X$ denotes $G_{i}$ and $w_{i}, X_{\circ}$ is the value when tip-off $\omega_{\text {tip-off }}=5^{\circ} / \mathrm{s} . G_{i 0}, w_{i 0}$ and $b_{j}$ are given in Table 2 .

\section{Simulation of the Orbit Generation and Track- ing Data Reduction for RSAT}

In SELENE project, the GEODYN II orbiting software will be used for data analysis and orbit determination. A simulation and data analysis research is done to compare the modified model with other ones. The force models adopted in this research are the same as which have been adopted by Kubo-oka and Sengoku (1999), except for the solar radiation models. The simulation method is also similar to their work.

Using different types of solar radiation pressure models, non tip-off, tip-off and cannonball mentioned earlier, the RSAT orbits are generated first from the same initial conditions. According to the current plan, the initial orbital elements of RSAT $a, e, i, \Omega, \omega, M$ are $3000 \mathrm{~km}, 0.38$, $90^{\circ}, 253^{\circ}, 133^{\circ}$ and $0^{\circ}$, respectively. The data sets of these generated orbits cover 30 days time interval from August 2 through September 1 in 2004. The integrating step or sampling rate is 10 seconds. Then, using these orbits data sets, the relative positions between the satellite and the center of the Moon, and the velocities of the satellite are compared at three dimentions at each sampling epoch. The magnitudes of different relative positions and of different velocities between the orbits of using non tip-off model and of using other models are shown in Fig. 4. It can be seen that when the tip-off is smaller than $5^{\circ} / \mathrm{s}$, the differences are less than $1.0 \mathrm{~m}$ or $1.0 \mathrm{~mm} / \mathrm{s}$ for the distance or speed, respectively. In all of other cases, correspondingly, the differences increase quickly to $10 \mathrm{~m}$ and $10 \mathrm{~mm} / \mathrm{s}$ in the first $2-3$ days after orbit insertion. The periodic pattern is similar due to the orbit period of RSAT. 

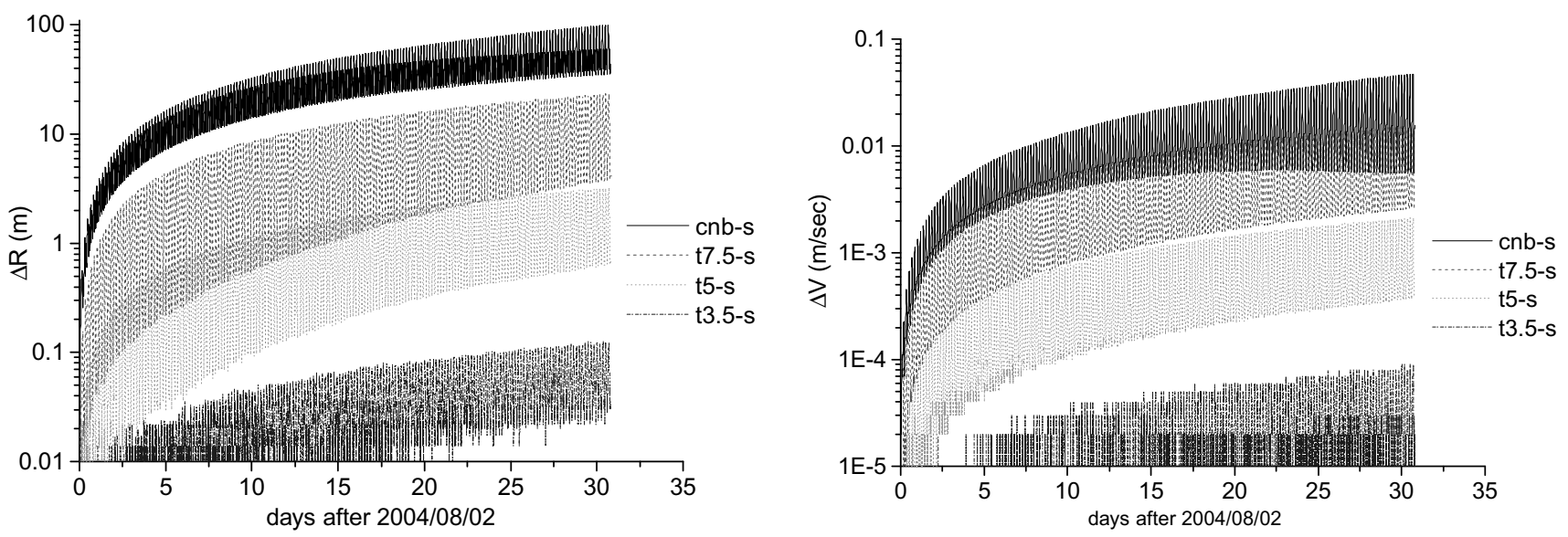

Fig. 4. Differences in orbit generation due to different types of solar radiation pressure model: cnb-cannonball, s-spin only (non tip-off), t-tip-off, numbers after $\mathrm{t}$ are tip-off values. $\Delta \mathrm{R}$, different position from the center of the Moon; $\Delta \mathrm{V}$, different velocity at same epoch, respectively.

Table 3. Post-fit RMS of the observation residuals.

\begin{tabular}{lccccc}
\multicolumn{7}{c}{ (Priori random noise RMS $=0.2 \mathrm{~mm} / \mathrm{s})$} \\
\hline tip-off Model & 0.0 & 3.0 & 5.0 & 7.5 & $10.0(\% \mathrm{~s})$ \\
\hline tip-off10 & 0.740 & 0.741 & 0.706 & & 0.201 \\
tip-off7.5 & 0.397 & 0.397 & & 0.200 & \\
tip-off5.0 & 0.204 & 0.205 & 0.200 & & 0.706 \\
tip-off3.0 & 0.200 & 0.200 & 0.205 & 0.397 & 0.741 \\
non tip-off & 0.200 & 0.200 & 0.205 & 0.398 & 0.741 \\
Cr 30 & 17.447 & 17.454 & 17.218 & 12.874 & 5.190 \\
Cr 15 & 2.048 & 2.047 & 2.337 & 1.973 & 1.847 \\
Cr 7 & 2.179 & 2.179 & 2.187 & 1.931 & 2.070 \\
Cr 2 & 2.285 & 2.283 & 1.927 & 2.285 & 3.141 \\
Cr 1 & 1.956 & 1.955 & 1.947 & 2.178 & 2.427 \\
\hline
\end{tabular}

non tip-off = Kubo-oka and Sengoku model.

tip-off $=$ upgraded model in this paper.

$\mathrm{Cr} n=$ coefficient of cannonball model, estimated every $n$ day.

Based on these orbits data sets, 2-way range-rate during the same time interval is simulated with the sampling rate of 20 seconds. In the simulation, only one tracking station (USUDA Tracking and Control Center of ISAS, Japan) is assumed. A random noise (RMS) of $0.2 \mathrm{~mm} / \mathrm{s}$ is added to the simulated data. This level of noise is the same as the expected RMS noise for RSAT range-rate tracking. Then, the simulated 2-way range-rate data is used to determine the orbit for RSAT. For each data set, length of the arc is taken to be 1 month, only the initial position and model sense velocity of the RSAT are estimated. Except for the solar radiation models, all of the forces models and the observation models are the same in both the orbit generation and the tracking data analysis. If the solar radiation models are also the same in them, RMS of the observation residuals should be equal or very close to RMS of the noise added to the tracking data. If the solar radiation models are different, the RMS probably should be different. However, if the RMS of the residual of different models are close to each other, this means the two types of solar radiation pressure models can be replaced by each other. The results of post-fit RMS of the observation residuals are given in Table 3 .

In Table 3, tracking data are simulated for 5 orbits which are separately generated by using the models of tip-off of $0.0,3.0,5.0,7.5$ and $10.0^{\circ} \mathrm{s}$. In the orbit determination, these models together with cannonball model are used for each data set to estimate the orbit separately. When cannonball model is used, the radiation pressure coefficient $\mathrm{Cr}$ at different frequency is also resolved along with the estimation of initial orbit state vector. Table 3 confirms the results drawn from Fig. 4. The difference between the solar radiation pressure models of a non tip-off RSAT and of a small tip-off $\left(\leq 5^{\circ} / \mathrm{s}\right)$ RSAT can be neglected. For a relatively large tip-off $\left(>5^{\circ} / \mathrm{s}\right)$, the difference increases quickly. It cannot be replaced by a non tip-off model. In an actual data analysis, this kind of information may give a chance to estimate the attitude of RSAT roughly. Although the RMS of the observation residuals tends to decrease when the radiation pressure coefficient being estimated more frequently, i.e. shorter than 2 weeks for each solution, it cannot be re- 
duced sufficiently. The difference between the cannonball model and the model of non tip-off have been discussed in great detail by Kubo-oka and Sengoku (1999). Their conclusions are also suitable for the difference between the cannonball model and the model of tip-off.

\section{Summary and Discussion}

Using the method introduced by Kubo-oka and Sengoku (1999), the solar radiation pressure acting on S/C can be analyzed for a spin stabilized $\mathrm{S} / \mathrm{C}$ without any tip-off or nutation. In the case of mixing spin with a small tip-off, by solving the Euler's equations for $\mathrm{S} / \mathrm{C}$ as a rigid body, the instantaneous and the mean solar radiation pressure acting on the $\mathrm{S} / \mathrm{C}$ can also be estimated and simulated. It is found that the difference of mean pressures between the cases of non tip-off and of a small tip-off is not large. Therefore model improvement can be achieved by adding a small correction term to the non tip-off model for one axis.

Assuming the shape of the satellite is an octagonal pillar, the solar radiation pressure model of RSAT and VSAT is modified by taking a small tip-off into account. Introducing different types of the models into the simulations of orbit generation and tracking data reduction separately, the difference and similarity between the results can be recognized. For a tip-off larger than $5^{\circ} / \mathrm{s}$, the observation residuals can be reduced by using the modified solar radiation pressure model compared to the non tip-off or to the standard cannonball modeling techniques. When a tip-off is smaller than $5 \% \mathrm{~s}$, the modified model can be replaced by using the non tip-off model in data analysis.

Compared to the 'cannonball' model, the models of both non tip-off and tip-off have a disadvantage in orbit determination. The attitude of the $\mathrm{S} / \mathrm{C}$ must be known together with other state information of the satellite. Acceleration due to the radiation pressure depends on these conditions strongly. Onboard RSAT or VSAT, there is not any active or passive attitude measuring and controlling system, so it is difficult to precisely know these conditions and parameters, such as the angle $\theta$ and the tip-off velocity. However, because the stabilized spin can keep the $\mathrm{S} / \mathrm{C}$ attitude for a long period after being separated from the main orbiter, a possible idea is to use the attitude information of RSAT or of VSAT at the epoch of separation as initial value; spin rates of them can be obtained precisely from the residuals of Doppler track- ing data; the tip-off at separation epoch can be measured by using a fast recording camera to record the separation process, like what has been done in BBM experiments. By this means, the angle $\theta$ and tip-off velocity may be set as variable parameters. A sub-program can be incorporated into the orbiting software so as to estimate them together with the orbit determination. Future work should investigate the possibility of realizing this operation. Also, a 4-way SST Doppler link for SELENE needs to be simulated to study how the solar radiation pressure acting on RSAT with a small tip-off will affect orbit determination of the main orbiter, and consequently, how it will affect the recovery of lunar gravity field.

Acknowledgments. Authors wish to express his gratitude to Drs. T. Kubo-oka of Communication Research Laboratory and R. Floberghagen of European Space Agency for their useful advice on this research work, and for their careful readings and helpful comments on this article. GEODYN II/SOLVE programs were introduced to NAO-J under a joint research contract with NASA/GSFC.

\section{References}

Floberghagen, R., P. Visser, and F. Weischede, Lunar albedo force modeling and its effect on low lunar orbit and gravity field determination, $A d v$. Space Res., 23, 733-738, 1999.

Kubo-oka, T. and A. Sengoku, Solar radiation pressure model for the relay satellite of SELENE, Earth Planets Space, 51, 979-986, 1999.

Lambeck, K., Geophysical Geodesy, pp. 284-289, Clarendon Press, Oxford, 1988 .

Macmillan, W. D., Dynamics of Rigid Body, pp. 181-186, Dover Pub. Inc., New York, 1936.

Milani, A., A. M. Nobili, and P. Farinella, Non-gravitational Perturbations and Satellite Geodesy, 125 pp., Universita di Pisa, Italy, 1987.

Namiki, N., H. Hanada, T. Tsubokawa, N. Kawano, M. Ooe, K. Heki, T. Iwata, M. Ogawa, T. Takano, and RSAT/VRAD/LALT mission groups (1999), Selenodetic experiments of SELENE: Relay subsatellite, differential VLBI, and laser altimeter, Adv. Space Res., 23, 1817-1820, 1999.

Pavlis, D. E., S. Luo, P. Dahiroc, J. J. McCarthy, and S. B. Luthke, GEODYN-II Operations Manual, 428 pp., GSFC of NASA, Greenbelt, Maryland, 1998.

Ping, J., Y. Kono, N. Kawano, and K. Matsumoto, How S/C tip-off and free nutation affect Doppler tracking in SELENE, J. Geod. Soc. Japan, 47(1), 576-582, 2001.

Sengoku, A., M. K. Cheng, and B. E. Schutz, Anisotropic reflection effect on satellite, Ajisai, J. Geodesy, 70, 140-145, 1995.

J. Ping (e-mail: jsping@miz.nao.ac.jp), A. Sengoku, N. Nagaoka, T. Iwata, K. Matsumoto, and N. Kawano 